\title{
Giant gravitons in the Schrödinger holography
}

\author{
George Georgiou $^{a}$ and Dimitrios Zoakos ${ }^{a, b}$ \\ ${ }^{a}$ Department of Physics, National and Kapodistrian University of Athens, \\ 15784 Athens, Greece \\ ${ }^{b}$ Hellenic American University, \\ 436 Amherst st, Nashua, NH 03063 U.S.A. \\ E-mail: ggeo@phys.uoa.gr, zoakos@gmail.com
}

ABSTRACT: We construct and study new giant graviton configurations in the framework of the non-supersymmetric Schrödinger holography. We confirm in the original Schrödinger spacetime, the picture discovered previously in the pp-wave limit of the geometry, namely that it is the giant graviton that becomes the energetically favored stable configuration compared to the point graviton one. Furthermore, there is a critical value of the deformation above which the point graviton disappears from the spectrum. The former fact leads also to the possibility of tunnelling from the point graviton to the giant graviton configuration. We calculate, explicitly, the instanton solution and its corresponding action which gives a measure of the tunnelling probability. Finally, we evaluate holographically the three-point correlation function of two giant gravitons and one dilaton mode as a function of the Schrödinger invariant.

Keywords: AdS-CFT Correspondence, Brane Dynamics in Gauge Theories, D-branes

ArXiv EPrint: 2010.03952 


\section{Contents}

1 Introduction 1

2 Giant gravitons in a Schrödinger geometry 3

3 Instanton transitions $\quad 7$

$\begin{array}{lll}3.1 & \text { Special instanton solution } & 10\end{array}$

4 Three-point function of two giant gravitons and a dilaton mode $\quad 13$

$\begin{array}{lll}5 & \text { Conclusions } & 16\end{array}$

\section{Introduction}

An important and efficient tool in exploring a gravity background (especially one that comes as a continuous deformation of the $\mathcal{N}=4 \mathrm{SYM}$ ) is the inclusion of brane probes and the subsequent study of their dynamics. A prominent example in this class of configurations is a D3-brane that wraps a three-sphere inside the five-sphere of the celebrated $A d S_{5} \times S^{5}$ geometry and it known as the giant graviton [1]. It has the same quantum numbers as the point particle and a nonzero angular momentum along the equator of the internal space. Stability against shrinking is guaranteed by the RR repulsion but also the perturbative stability analysis around the giant graviton solution has been performed in [2]. When the three-sphere that the giant wraps is inside the AdS part of the space the configuration to be realized is the dual giant graviton, with similar properties [3, 4].

While in the original $A d S_{5} \times S^{5}$ geometry (and depending on the value of the conjugate angular momentum) the point graviton is either energetically favored or at most degenerate with respect to the giant graviton, the situation becomes intricate and interesting when the geometry gets deformed. When the deformation is purely on a sphere, as in the marginally deformed backgrounds, there is a variety of behaviors but in none of them the energetically favored solution is the giant graviton. More specifically, there are cases that the degeneracy that is inherited by the parent undeformed background is intact $[5,6]$, cases that the degeneracy is lifted in favor of the point graviton $[7,8]$ and cases where the initially energetically favored point graviton becomes even more pronounced [7]. However, when the deformation involves directions of the anti de Sitter space, as it happens in the cases of the non-commutative or the dipole deformation, there are situations where the energetically favored solution is the giant graviton (see [9] for the non-commutative example and [10] for the pp-wave geometry of certain dipole deformed theories).

There are several interesting examples of gauge/gravity dualities in which the original AdS/CFT scenario [11] is deformed. One such example is the duality between a certain spacetime with Schrödinger symmetry and its dual dipole-deformed non-relativistic 
conformal field theory [12]. Compared to the original AdS/CFT duality very few observables have been calculated in the Schrödinger/dipole CFT version of the correspondence. In particular, in [13] and [14] two and three, as well as $n$-point correlation functions of scalar operators were calculated by employing the gravity side of the correspondence. It is important to mention that all the operators involved in these correlators correspond to point-like strings propagating in the $S c h_{5} \times S^{5}$ background. In [15] extended dyonic giant magnon and spike solutions were constructed and their dispersion relations were derived. ${ }^{1}$ A complementary study with finite size corrections of those classical solutions was presented in [17]. ${ }^{2}$ The existence of the aforementioned solutions is in complete agreement with the fact that the theories involved in the Schrödinger/dipole CFT duality remain integrable despite the presence of the deformation. In the same work [15] an exact, in the 't Hooft coupling $\lambda$, expression for the dimensions of certain gauge invariant operators dual to the giant magnons was conjectured. Subsequently, this conjecture was further improved in [20] in such a way that it is in perfect agreement with the string spectrum in the ppwave limit of the Schrödinger spacetime. Furthermore, agreement between this expression and the one-loop anomalous dimension of BMN-like operators was found in the large $J$ limit providing, thus, further evidence in favor of the correspondence. On the field theory side, the one-loop spectrum of operators belonging in a SL(2) closed sub-sector of the null dipole-deformed CFT has been studied in [21]. The authors found agreement between the one-loop anomalous dimensions of certain long operators and the energies of the dual string solutions (see [22], as well).

Subsequently, by utilising the Schrödinger background, the three-point functions involving two heavy and one light operator were calculated holographically in [23]. The heavy states were generalizations either of the giant magnon or spike solutions constructed in [15] while the light operator was chosen to be one of the dilaton modes. These results for the three-point functions are the first in the literature in which the heavy states participating in three-point correlation functions are described by extended string solutions. The aforementioned results are in complete agreement with the form of the correlator dictated by non-relativistic conformal invariance and provide us with the leading term of the correlators in the large $\lambda$ expansion. Finally, pulsating strings solutions in the Schrödinger background were recently derived in $[24,25]$ while the holographic Fisher information metric in Schrödinger spacetime was studied in [26].

Very recently, giant graviton solutions in the pp-wave limit of the Schrödinger geometry were constructed in [10]. These solutions have the remarkable property that the deformation breaks the degeneracy between the point and giant graviton solutions in favour of the giant graviton which becomes the energetically favoured stable solution. The focus of the current paper is twofold. On one hand, it is to identify the classical configurations describing giant gravitons in the Schrödinger background before taking the pp-wave limit and on the other to use these solutions in order to probe the holography in Schrödinger spacetimes.

\footnotetext{
${ }^{1}$ Giant magnon solutions in other deformed geometries like the $\beta$-deformed theories have been found in $[16]$.

${ }^{2}$ Giant magnon-like solutions with a different dispersion relation were found in [18], while giant magnons and spiky strings on $S c h_{5} \times T^{1,1}$ and the corresponding dispersion relations were found in [19].
} 
This second goal will be accomplished through the calculation of the three-point correlation functions of the dilaton modes and two "heavy" operators. The giant gravitons will serve as the gravity duals of the heavy states and the result of the holographic computation will provide us with the leading term in the large $\lambda$ expansion of the three-point correlators.

The plan of the paper is as follows: in section 2, we revisit the type IIB supergravity solution of the Schrödinger background and present a consistent giant graviton ansatz. The Hamiltonian of the system is a function of two variables: the deformation parameter and the conjugate momentum with respect to one of the internal angles of the five-sphere. We investigate the behavior of the system either fixing the conjugate momentum and varying the deformation parameter or vice versa and extract a plethora of interesting observations. The importance of the ansatz we introduce is that the zero deformation limit of our Hamiltonian leads smoothly to the Hamiltonian of the undeformed $A d S_{5} \times S^{5}$ that appears in $[1,3]$. In section 3 we focus the attention to potential tunnelling effects between the two graviton solutions and calculate instanton transitions, in that part of the parametric space where the giant graviton is the energetically favored solution. Emphasis is put on a novel instanton solution that arrises when the deformation parameter and the conjugate momentum are related through a constraint in such a way that, despite the presence of a deformation, the energies of the two gravitons, the point and the giant graviton, are equal. In section 4, we compute holographically the three-point correlation function of the dilaton modes and two "heavy" operators whose gravity duals are the giant gravitons, in the special case where the giant and the point graviton are degenerate. The result is in complete agreement with the constraints put by conformal invariance. In section 5 we conclude the paper and identify potential future directions for endeavors in the framework of Schrödinger holography.

\section{Giant gravitons in a Schrödinger geometry}

The starting point for the analysis we will present in this paper is the expressions for the supergravity solution of the $10 \mathrm{~d} S c h_{5} \times S^{5}$ geometry in global coordinates [21, 27]

$$
\begin{aligned}
& \frac{d s^{2}}{L^{2}}=-\left(1+\frac{\mu^{2}}{Z^{4}}\right) d T^{2}+\frac{1}{Z^{2}}\left(2 d T d V+d Z^{2}+d \vec{X}^{2}-\vec{X}^{2} d T^{2}\right)+d s_{S^{5}}^{2} \\
& d s_{\mathrm{S}^{5}}^{2}=(d \chi+\omega)^{2}+d s_{\mathbb{C P}^{2}}^{2} \quad \& \quad d s_{\mathbb{C P}^{2}}^{2}=\frac{d \rho^{2}}{1-\rho^{2}}+\rho^{2}\left[\Sigma_{1}^{2}+\Sigma_{2}^{2}+\left(1-\rho^{2}\right) \Sigma_{3}^{2}\right]
\end{aligned}
$$

where we have written the metric of the five-sphere as an $S^{1}$-fibration over the $\mathbb{C P}^{2}$ and the quantities $\Sigma_{1}, \Sigma_{2}$ and $\Sigma_{3}$ are defined as follows

$$
\begin{array}{rlrl}
\Sigma_{1} & \equiv \frac{1}{2}(\cos \psi d \theta-\sin \psi \sin \theta d \phi) & \Sigma_{2} & \equiv \frac{1}{2}(\sin \psi d \theta+\cos \psi \sin \theta d \phi) \\
\Sigma_{3} & \equiv \frac{1}{2}(d \psi-\cos \theta d \phi) \quad \text { and } \quad \omega & \equiv \rho^{2} \Sigma_{3} .
\end{array}
$$

The metric is accompanied with a magnetic field

$$
\frac{B_{2}}{L^{2}}=\frac{\mu}{Z^{2}} d T \wedge(d \chi+\omega)
$$


and a RR five-form

$$
\frac{F_{5}}{L^{4}}=\frac{4}{Z^{5}} d T \wedge d V \wedge d \vec{X} \wedge d Z+4 \rho^{3} d \rho \wedge \Sigma_{1} \wedge \Sigma_{2} \wedge \Sigma_{3} \wedge d \chi .
$$

In order to describe Giant Graviton solutions in the Schrödinger background (2.1), we have to consider the action of a probe D3-brane. This action consists of the sum of the Dirac-Born-Infeld (DBI) term and of the Wess-Zumino (WZ) term

$$
S_{\mathrm{D} 3}=-T_{3} \int d^{4} \xi e^{-\Phi} \sqrt{-\operatorname{det} \mathcal{P}\left[g+B+2 \pi \alpha^{\prime} F\right]}+T_{3} \int \sum_{q} \mathcal{P}\left[A_{q} \wedge e^{B+2 \pi \alpha^{\prime} F}\right]
$$

where $\mathcal{P}$ denotes the pullback of the different spacetime fields on the brane worldvolume and $T_{3}$ is the tension of the D3-brane.

We consider a D3-brane probe that extends along the following directions

$$
\xi_{0}=\tau, \quad \xi_{1}=\theta, \quad \xi_{2}=\phi, \quad \xi_{3}=\psi
$$

and the ansatz for the rest of the coordinates is as follows

$$
T=\kappa \tau, \quad V=\nu \tau, \quad \vec{X}=0, \quad Z=Z_{0}, \quad \rho=\frac{\rho_{0}}{L}, \quad \chi=\omega \tau .
$$

Notice that $\rho_{0}$ is the size of the graviton in the internal space.

Substituting the ansatz (2.7) in (2.5) and integrating the spatial coordinates of the world volume, we obtain the following expressions for the on-shell action and the Lagrangian

$$
S_{\mathrm{D} 3}=\int d \tau L_{\mathrm{D} 3} \quad \text { with } \quad L_{\mathrm{D} 3}=-\frac{N}{L^{4}}\left[\rho_{0}^{3} \sqrt{1+\Gamma-\left(L^{2}-\rho_{0}^{2}\right)\left(\omega^{2}-\frac{\Delta^{2}}{L^{2}}\right)}-\rho_{0}^{4} \omega\right]
$$

where, to simplify notation, we have introduced the following definitions of constants

$$
\kappa=\frac{1}{L}, \quad \Gamma=-\frac{2 L}{Z_{0}^{2}} \nu, \quad \Delta=\frac{\mu}{Z_{0}^{2}}, \quad 2 \pi^{2} T_{3}=\frac{N}{L^{4}} .
$$

The conjugate momenta to $\chi$ and $V$ are given by

$$
J_{1}=\frac{\partial L_{\mathrm{D} 3}}{\partial \omega}, \quad J_{2}=\frac{\partial L_{\mathrm{D} 3}}{\partial \Gamma} .
$$

An important comment is in order. In contradistinction to the giant graviton ansatz in the pp-wave Schrödinger geometry [10], in the current case there are three conserved quantities (namely $E, J_{1}$ and $J_{2}$ ) instead of two ( $E$ and $J_{1}$ in the pp-wave case). The need for a generalization of the previous giant graviton ansatz is coming for the following reason. It is known that the ansatz (2.7) satisfies the equations of motion only at the extrema of the on-shell action (see also the discussion in [10]). Following this reasoning, we calculated the equations of motion for the coordinates that are orthogonal to the brane worldvolume directions (namely $T, V, \vec{X}, Z, \rho$ and $\chi$ ) and indeed we realized that the equations of motion for $Z$ and $\rho$ are not automatically satisfied. The equation of motion for $\rho$ is satisfied when we impose that the derivative of the Lagrangian with respect to $\rho$ vanishes. However, the 
equation of motion for $Z$ is not satisfied unless we fix the value of $J_{2}$. Turning on an extra coordinate dependence in the giant graviton ansatz (that for $V$ ) is necessary and gives us the extra freedom that is needed in order for all the equations of motion to be satisfied at the extrema of the Lagrangian. For this to happen the conjugate momentum $J_{2}$ should acquire the following value

$$
J_{2}=\frac{N}{2 L} \frac{V\left(\rho_{0}\right)}{\sqrt{1-\Delta^{2}\left(1-\frac{\rho_{0}^{2}}{L^{2}}\right)}} \quad \text { with } \quad\left(V\left(\rho_{0}\right)\right)^{2}=\frac{\rho_{0}^{6}}{L^{6}}+\frac{\left(\frac{J_{1}}{N}-\frac{\rho_{0}^{4}}{L^{4}}\right)^{2}}{1-\frac{\rho_{0}^{2}}{L^{2}}} .
$$

The corresponding Hamiltonian $H=J_{1} \omega+J_{2} \Gamma-L_{\mathrm{D} 3}$ for the giant graviton solution in the Schrödinger background becomes

$$
H=\frac{N}{L} \frac{V\left(\rho_{0}\right)}{\sqrt{1-\Delta^{2}\left(1-\frac{\rho_{0}^{2}}{L^{2}}\right)}} .
$$

Taking the limit of zero deformation (i.e. $\Delta \rightarrow 0$ ) the denominator becomes the unit and we recover the Hamiltonian of the $\mathcal{N}=4$ giant graviton on the sphere $[1,3,4]$. The quantity inside the square root in the denominator of (2.12) should be always positive, and this sets an upper bound for the value of $\Delta$. The range of values for the deformation parameter is

$$
0 \leq \Delta \leq\left(1-\frac{\rho_{0}^{2}}{L^{2}}\right)^{-1 / 2}
$$

In figure 1 we plot the Hamiltonian (2.12) as a function of the graviton's size $\rho_{0}$, for different values of the deformation $\Delta$ and of the conjugate momentum $J_{1}{ }^{3}$ In all expressions and figures below we have chosen units such that the radius of the 5 -sphere is $L=1$ and we have also set $N=1$, that is whereever one sees $J_{1}$ it is $J_{1} / N$ that should appear. On the left plot, we fix $J_{1}$ to a value that is less than one (more specifically $\left.J_{1}=0.9\right)$ and increase the value of the deformation parameter $\Delta$. For $\Delta=0$ we recover the $\mathcal{N}=4$ result, where the energy of the point and of the giant graviton are the same. Turning on the deformation parameter the initial degeneracy between the three extrema disappears and remarkably, the energetically favored solution (the one having less energy) is the giant graviton. This behavior is in qualitative agreement with the giant graviton calculation in the pp-wave Schrödinger background [10]. Keep increasing the value of $\Delta$, we will continue having three extrema, but the extremum at the position $\rho_{0}=0$ will change from a minimum to a maximum at the value $\Delta=1 / \sqrt{2}$. At exactly this value for $\Delta$ the second derivative of the Hamiltonian (2.12) with respect to $\rho_{0}$ at $\rho_{0}=0$ will change sign from positive to negative and if one keeps increasing the deformation parameter there is a value of $\Delta=1$ above which the point graviton will cease to exist. Notice that the behavior

\footnotetext{
${ }^{3}$ We follow the presentation style that was introduced in [3] and allow $\rho_{0}$ to take negative values. The range of negative values corresponds to the D3-brane expanding into the five-sphere with the angular coordinate having the opposite orientation. Since the potential (2.12) is even under $\rho_{0} \rightarrow-\rho_{0}$ the negative $\rho_{0}$ configuration is identical to the positive $\rho_{0}$ configuration.
} 

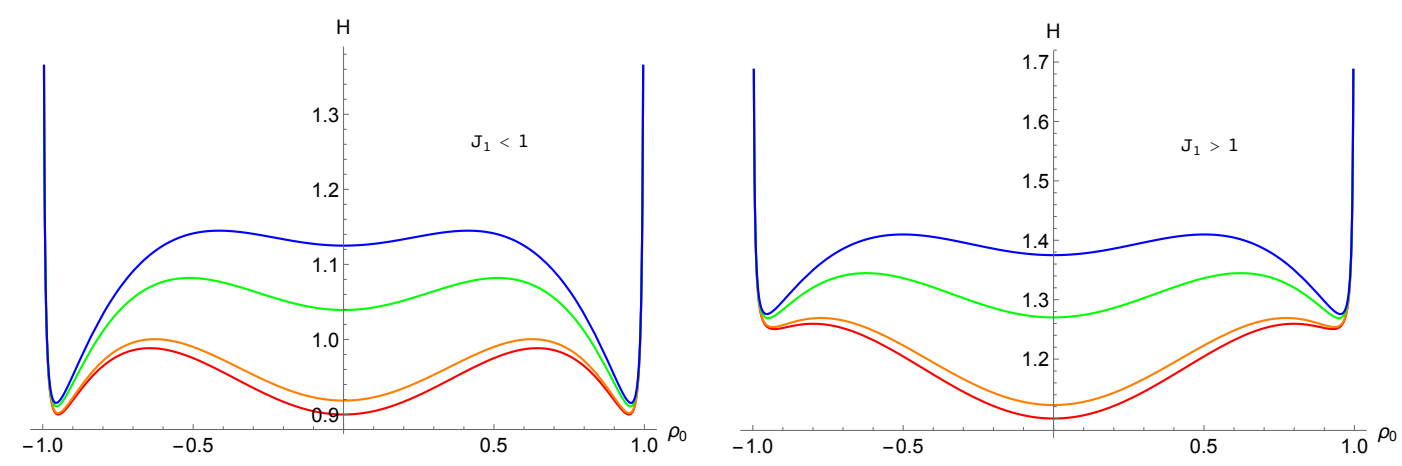

Figure 1. Hamiltonian (2.12) as a function of the graviton's size $\rho_{0}$, for different values of the deformation $\Delta$. The left (right) plot is for values of $J_{1}<1\left(J_{1}>1\right)$ and in units $N=L=1$. The correspondence between color and values of $\Delta$ for both plots of this figure is Red $\Rightarrow \Delta=0$, Orange $\Rightarrow \Delta=0.2$, Green $\Rightarrow \Delta=0.5 \&$ Blue $\Rightarrow \Delta=0.6$. For both graphs when $\Delta>1 / \sqrt{2}$ the point graviton becomes a maximum and the potential acquires the shape of a Mexican-hat with the giant gravitons being the only minima. If we reinstate units the vertical axis is $H L / N$, the horizontal axis is $\rho_{0} / L$ and the plots are for different values of $J_{1} / N$.

of the Hamiltonian as a function of the graviton's size $\rho_{0}$ will remain qualitatively unaltered if we change the value of $J_{1}$, as long as we stay in the range of $J_{1}<1$.

On the right plot of figure 1, we fix $J_{1}$ to a value that is above one (more specifically $J_{1}=1.1$ ) and increase the value of the deformation parameter $\Delta$. For $\Delta=0$ we have three extrema and the extremum at $\rho_{0}=0$ is the energetically favored solution, which is the $\mathcal{N}=4$ result. Increasing the value of $\Delta$, increases the value of the energy at the $\rho_{0}=0$ extremum and decreases the values of the other two extrema. As a result of this, there is a critical value of the deformation parameter $\Delta$ for which the three extrema have the same energy. This value depends on the value of $J_{1}$ and in figure 6 we depict the relation between the critical $\Delta$ and $J_{1}$. In the next section we will analyze in detail this special class of solutions. In this region of $\Delta<\Delta_{\text {crit }}$ the point graviton solution is energetically favored with respect to the giant graviton solution. The behavior of the Hamiltonian for values of the deformation parameter $\Delta$ above the critical value is identical to the behavior we already described for $J_{1}<1$. More specifically, the giant graviton becomes the energetically favored solution and for values above $\Delta=1 / \sqrt{2}$ the extremum at the point graviton position turns from a minimum to a maximum and for $\Delta>1$ the point graviton is not any more part of the spectrum (see also the discussion in [10]).

Instead of fixing the conjugate momentum $J_{1}$ and varying the deformation parameter $\Delta$, we could have done the inverse. Such an analysis shows that keeping the value of the deformation parameter fixed and below $\Delta=1 / \sqrt{2}$, that is in the region where the point graviton is still a minimum, and increasing the value of the conjugate momentum beyond $J_{1}=1$, there is a critical value of $J_{1}$ above which the giant graviton solution completely disappears. ${ }^{4}$ At this value of $J_{1}$ both the first and the second derivative of the Hamiltonian

\footnotetext{
${ }^{4}$ Notice that this happens only for values $J_{1}>1.125$. For $J_{1} \leq 1.125$ the giant graviton solutions are always present, as metastable states at worst.
} 


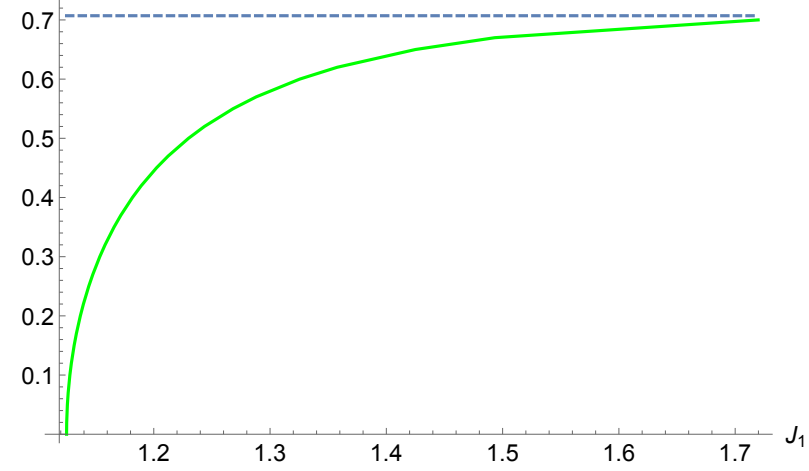

Figure 2. Plot of the conjugate momentum $J_{1}$ for different values of the deformation parameter $\Delta$ (all of them below the value $\Delta=1 / \sqrt{2}$ ). Above the green line there is a giant graviton solution while below the green line there is only the point graviton solution.

with respect to $\rho_{0}$ at the giant graviton position vanish. In figure 2 we have plotted this critical value of $J_{1}$ as a function of the deformation parameter $\Delta$. In the undeformed $\mathcal{N}=4$ case, the critical value of the conjugate momentum is $J_{1}=1.125$ [3]. Notice that if the value of the deformation parameter is above $\Delta=1 / \sqrt{2}$ then the extrema corresponding to the giant graviton solution never disappear. This is related to the fact that for values of the deformation parameter above $\Delta=1 / \sqrt{2}$ the extremum at the point graviton position turns from a minimum to a maximum.

\section{Instanton transitions}

The analysis of the previous section has uncovered two brane configurations that in the framework of a Schrödinger background describe a graviton state with angular momentum $J_{1}$ : from one side it is the point-like graviton and from the other side the giant graviton, a configuration that consists of a spherical D3-brane extending into the five-sphere. Interestingly, even though the five-sphere is not deformed in the Schrödinger deformation of the parent $A d S_{5} \times S^{5}$ background we consider, due to the presence of the magnetic field, the Hamiltonian of the giant graviton depends on the deformation parameter in a non-trivial way. The interplay between the conjugate momentum $J_{1}$ and the deformation parameter $\Delta$ creates a rich space of parameters that we analytically examined in the previous section.

Quantum mechanically we expect the two graviton states to mix and motivated from this expectation we are looking for instanton solutions that tunnel from one graviton state to the other. In the current section we will derive explicit expressions for the instanton solutions that evolve between the expanded three-brane state and the zero-size state. In particular, we will be mostly interested in the tunnelling from the point graviton to the giant graviton configuration since, as was discussed in the previous section, the effect of the deformation $\mu$ or equivalently $\Delta$ is to make the giant graviton the energetically favorable state.

To begin the analysis we extend the ansatz that we introduced in (2.7) to allow for a time-dependent $\rho_{0}$ coordinate, that is to allow for a time-dependent size of the graviton. 
The Lagrangian is now extended to the following expression

$$
L_{\mathrm{D} 3}^{\mathrm{E}}=-\frac{N}{L^{4}}\left[\rho_{0}^{3} \sqrt{1+\Gamma-\left(L^{2}-\rho_{0}^{2}\right)\left(\omega^{2}-\frac{\Delta^{2}}{L^{2}}\right)+\frac{\dot{\rho}_{0}^{2}}{1-\frac{\rho_{0}^{2}}{L^{2}}}}-\rho_{0}^{4} \omega\right]
$$

where we have analytically continued to euclidean time $\tau \rightarrow i \tau$. This results in an inversion with respect to the $\rho_{0}$-axis of the potential energy of figure 1 .

As a next step we perform a Legendre transformation to eliminate $\omega$ and $\Gamma$ in favor of the conserved angular momenta $J_{1}$ and $J_{2}$ and produce the Routhian

$$
\mathcal{R}^{\mathrm{E}}=\omega \frac{\partial L_{\mathrm{D} 3}^{\mathrm{E}}}{\partial \omega}+\Gamma \frac{\partial L_{\mathrm{D} 3}^{\mathrm{E}}}{\partial \Gamma}-L_{\mathrm{D} 3}^{\mathrm{E}} .
$$

Instead of working with the second order equations of motion coming from the Euclidean action (3.1) (or the Routhian (3.2)), we determine the instanton solution by the evaluation of the corresponding conserved energy

$$
H^{\mathrm{E}}=\dot{\rho}_{0} \frac{\partial \mathcal{R}^{\mathrm{E}}}{\partial \dot{\rho}_{0}}-\mathcal{R}^{\mathrm{E}}=p
$$

Following the same reasoning we have already discussed in detail in section 2, we calculate from the full DBI plus WZW Lagrangian the equations of motion for the coordinates that are orthogonal to the brane worldvolume directions. Using the expression for the derivative of $\rho_{0}$ with respect to $\tau$ (i.e. $\dot{\rho}_{0}$ ) that is obtained from (3.3), all the equations of motion are satisfied except from the equation of motion for $Z$. That equation provides a constraint between the conjugate momentum $J_{2}$ and the conserved energy $p$, namely

$$
2 J_{2}+p=0 \Rightarrow J_{2}=\frac{N}{2 L} \frac{V\left(\rho_{0}\right)}{\sqrt{1-\Delta^{2}\left(1-\frac{\rho_{0}^{2}}{L^{2}}\right)+\frac{\dot{\rho}_{0}^{2}}{1-\frac{\rho_{0}^{2}}{L^{2}}}}} .
$$

In the last equation $V\left(\rho_{0}\right)$ is the same function defined in (2.11). One should, however, keep in mind that in (3.4) $\rho_{0}$ is a function of the world-volume time $\tau$ while in (2.11) $\rho_{0}$ is a constant.

Substituting (3.4) in (3.2) we obtain the expression for the Routhian

$$
\mathcal{R}^{\mathrm{E}}=\frac{N}{L} \frac{V\left(\rho_{0}\right)}{\sqrt{1-\Delta^{2}\left(1-\frac{\rho_{0}^{2}}{L^{2}}\right)+\frac{\dot{\rho}_{0}^{2}}{1-\frac{\rho_{0}^{2}}{L^{2}}}}}\left(1+\frac{\dot{\rho}_{0}^{2}}{1-\frac{\rho_{0}^{2}}{L^{2}}}\right)
$$

while substituting (3.4) in (3.3) we obtain the expression for the Hamiltonian

$$
H^{\mathrm{E}}=-\frac{N}{L} \frac{V\left(\rho_{0}\right)}{\sqrt{1-\Delta^{2}\left(1-\frac{\rho_{0}^{2}}{L^{2}}\right)+\frac{\dot{\rho}_{0}^{2}}{1-\frac{\rho_{0}^{2}}{L^{2}}}}} .
$$

Notice that setting to zero the value of the deformation parameter $\Delta$ the expressions (3.5) and (3.6), for the Routhian and the Hamiltonian respectively, flow to the undeformed counterparts in [3]. 
For the remainder of the current section we will focus on cases where the giant graviton is the energetically favored solution and we will construct instantons that describe tunnelling from the point to the giant graviton configuration. In terms of the discussion we detailed in the previous section around figure 1, there are two cases of interest:

- $J_{1}<1$ and $0<\Delta<1 / \sqrt{2}$ (left plot of figure 1 ). We remind the reader that for $\Delta>1 / \sqrt{2}$ the point graviton is not any more part of the spectrum.

- $J_{1}>1$ and $\Delta_{\text {crit }}<\Delta<1 / \sqrt{2}$ (right plot of figure 1 ).

To proceed the analysis we set $L=N=1$ and since the point graviton at $\rho_{0}=0$ will be the instanton starting and ending point, the conserved energy obtains the following value

$$
H^{\mathrm{E}}\left(\rho_{0}\right)=H^{\mathrm{E}}\left(\rho_{0}=0\right)=-\frac{J_{1}}{\sqrt{1-\Delta^{2}}} .
$$

Solving the differential equation that arises from (3.6) with the boundary condition (3.7), we arrive at the following instanton solution

$$
\rho_{0}(\tau)=\left[\frac{J_{1}\left(1-2 \Delta^{2}\right)}{1+e^{2\left(\tau-\tau_{0}\right) \sqrt{1-2 \Delta^{2}}}-\left(1+\frac{J_{1}}{2}\right) \Delta^{2}+\frac{1}{16} \Delta^{2} e^{-2\left(\tau-\tau_{0}\right) \sqrt{1-2 \Delta^{2}} \mathcal{W}}}\right]^{1 / 2}
$$

where the auxiliary quantity $\mathcal{W}$ is defined as follows

$$
\mathcal{W}=J_{1}^{2} \Delta^{2}+4\left(1-J_{1}\right)\left(1-\Delta^{2}\right) .
$$

The constant $\tau_{0}$ that appears in (3.8) is an integration constant that gives the position of the instanton in euclidean time, it is in fact the zero mode that determines the instanton position. In the rest of the analysis and for all the plots we fix this constant to zero. i.e. $\tau_{0}=0$. In figure 3 we plot the instanton solution for two values of the conjugate momentum $J_{1}$, one less than one and one larger than one, and three different values of the deformation parameter $\Delta$.

The next step is the calculation of the euclidean action. Since the Routhian $\mathcal{R}^{\mathrm{E}}$ (3.5) evaluated around the point graviton position remains finite the evaluation of the integral $\int d \tau \mathcal{R}^{\mathrm{E}}$ diverges. To regularize the integral we subtract the Routhian at the point graviton extremum and the relevant quantity to calculate is the following

$$
\mathcal{S}=\int_{-\infty}^{\tau_{\text {turn }}} d \tau \Delta \mathcal{R} \quad \text { with } \quad \Delta \mathcal{R}=\mathcal{R}\left(\rho_{0}\right)-\mathcal{R}\left(\rho_{0}=0\right)
$$

where $\tau_{\text {turn }}$ is the time that corresponds to the maximum of the curve that is depicted in figure 3. Notice that the upper limit of integration is the turning point $\tau_{\text {turn }}$ since if the graviton manages to tunnel up to this point then it will immediately roll to the global minimum of the potential which is the giant graviton state. In figures 4 and 5 we have plotted the total action (only numerical evaluation of the integral in (3.10) is possible) as a function of the conjugate momentum $J_{1}$ and the deformation parameter $\Delta$. Calculation of the total action provides a measure for the degree of tunnelling. More 

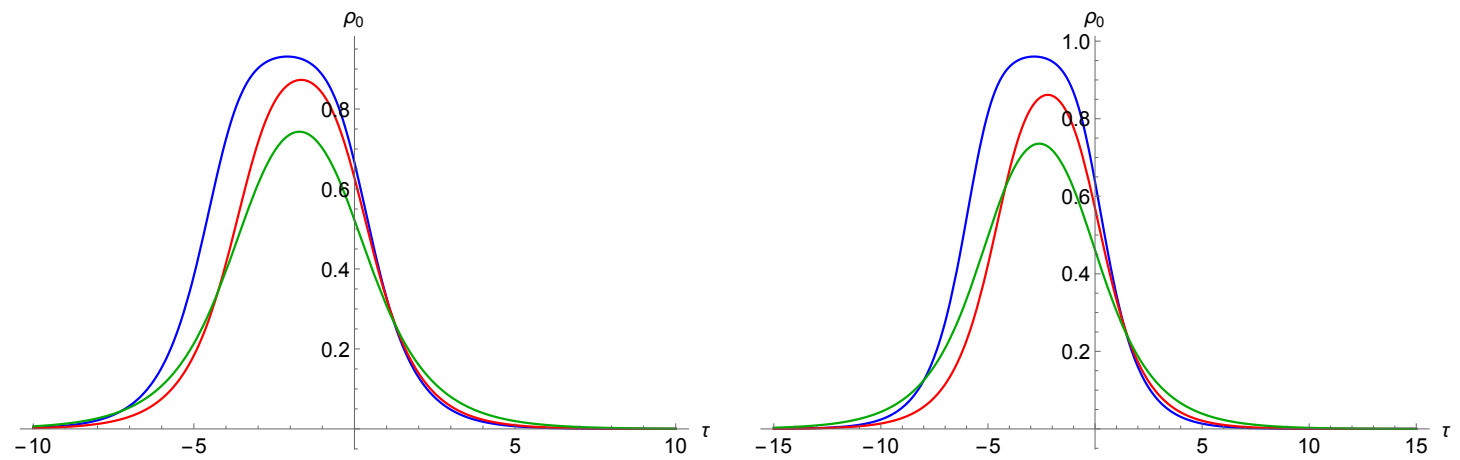

Figure 3. Plots of the instanton solution as a function of the euclidean time from equation (3.8). In terms of the potential that is depicted in figure 1, the instanton transition is from the point graviton extremum at $\tau \rightarrow-\infty$ to a position of the potential with the same energy as the point graviton at an intermediate time (where the maximum of the curve is located) and then back to the initial point graviton extremum at $\tau \rightarrow+\infty$. On the left plot the conjugate momentum is fixed to the value $J_{1}=0.9$ and the three curves correspond to different values of the deformation parameter: Blue $\Rightarrow \Delta=0.1$, Red $\Rightarrow \Delta=0.3 \&$ Green $\Rightarrow \Delta=0.5$. On the right plot the conjugate momentum is fixed to the value $J_{1}=1.05$ and the three curves correspond to different values of the deformation parameter: Blue $\Rightarrow \Delta=0.4$, Red $\Rightarrow \Delta=0.5 \&$ Green $\Rightarrow \Delta=0.6$. Notice that in the right plot the values of the deformation parameter are always above the critical value so that the giant graviton has less energy than the point graviton. For $J_{1}=1.05$ the critical value for the deformation is $\Delta_{\text {crit }} \approx 0.39$.

precisely, the transition probability from the point graviton to the stable giant graviton is proportional to the exponential of minus the action of (3.10). Two comments are in order. Firstly, notice that the transition probability is exponentially small since if we reinstate the number of colours $N$, which has been set to unity in the numerical calculation above, one gets $P \sim e^{-N s}$, where $s>0$. Secondly, note that figure 4 implies that as the deformation parameter increases the transition probability increases since $\mathcal{S}$ becomes smaller. This is fully consistent with the shape of the potential depicted in figure 1. It is evident from that figure that as the deformation parameter increases the height of the barrier which the brane has to tunnel through becomes smaller making the tunnelling more probable.

\subsection{Special instanton solution}

In this subsection, we will examine a special class of instanton solutions among those that are described by the analytic expression that is presented in (3.8). Setting to zero the value of the auxiliary parameter $\mathcal{W}$ that is defined in (3.9) introduces a constraint between the conjugate momentum $J_{1}$ and the deformation parameter $\Delta$. Solving the constraint with respect to $\Delta$ means that by choosing the value of $J_{1}$ we automatically fix the value of the deformation parameter $\Delta$ as follows ${ }^{5}$

$$
\mathcal{W}=0 \Rightarrow \Delta=\left[1-\frac{1}{4 N} \frac{J_{1}^{2}}{N-J_{1}}\right]^{-1 / 2} .
$$

In figure 6 we plot this relation between $\Delta$ and $J_{1}$.

\footnotetext{
${ }^{5}$ In all the expressions of this subsection we reinstate units by restoring $L$ and $N$.
} 

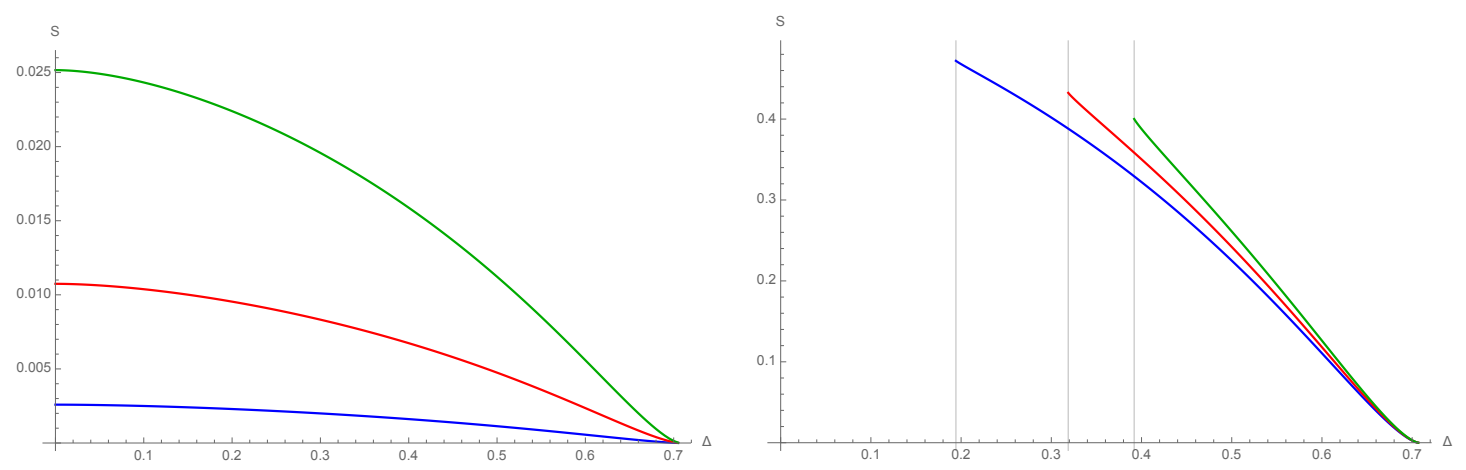

Figure 4. Total action as a function of the deformation parameter $\Delta$ for values of $J_{1}<1$ (left plot) and for values $J_{1}>1$ (right plot). When $J_{1}<1, \Delta$ takes all the values between 0 and $1 / \sqrt{2}$, while for $J_{1}>1 \Delta$ is restricted to the values that are above the red line in figure 6 . The gridlines emphasize those values, that are the lower limits for $\Delta$ given a value for $J_{1}$. The correspondence between colors and values of $J_{1}$ for the left plot is: Blue $\Rightarrow J_{1}=0.1$, Red $\Rightarrow J_{1}=0.2$ \& Green $\Rightarrow J_{1}=0.3$. The correspondence between colors and values of $J_{1}$ for the right plot is: Blue $\Rightarrow$ $J_{1}=1.01$, Red $\Rightarrow J_{1}=1.03 \&$ Green $\Rightarrow J_{1}=1.05$.

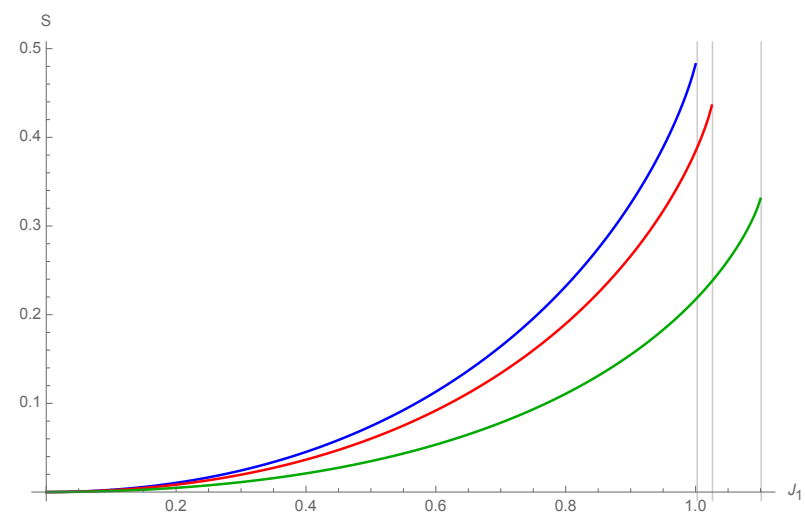

Figure 5. Total action as a function of $J_{1}$ for different values of $\Delta$. The upper bound for the value of $J_{1}$ depends on $\Delta$, as it is depicted in figure $6 . J_{1}$ should be always above the red line and the gridlines emphasize the maximum values for $J_{1}$. The correspondence between colors and values of $\Delta$ is: Blue $\Rightarrow \Delta=0.1$, Red $\Rightarrow \Delta=0.3 \&$ Green $\Rightarrow \Delta=0.5$.

Implementing the constraint (3.11) on the general instanton solution of (3.8) and calculating the limits at $\tau \rightarrow-\infty$ and $\tau \rightarrow+\infty$ we obtain the following results

$$
\frac{\rho_{0}}{L} \rightarrow 0 \quad \text { for } \quad \tau \rightarrow-\infty \quad \& \quad \frac{\rho_{0}}{L} \rightarrow \sqrt{2-\frac{J_{1}}{N}} \text { for } \tau \rightarrow+\infty .
$$

The last two relations fully determines the range of values for both $J_{1}$ and $\Delta$. More specifically, we have that $\Delta \in[0,1 / \sqrt{2})$ and $J_{1} / N \in[1,2)$. On the left plot of figure 7 the special instanton solution for $\mathcal{W}=0$ is presented. It corresponds to an instanton transition from the zero-size point graviton solution at $\tau \rightarrow-\infty$ to the expanded giant graviton solution at $\tau \rightarrow+\infty$. Notice that another instanton solution, symmetric with respect to the vertical axis at $\tau=0$, exists that would correspond to a transition from the 


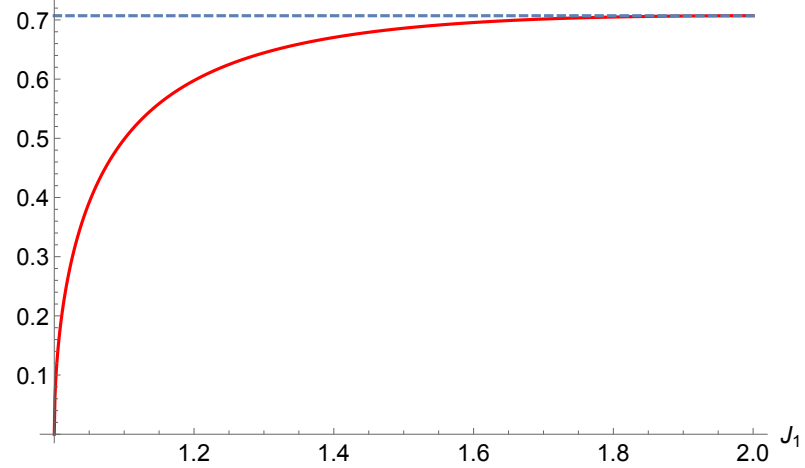

Figure 6. Plot of the relation between $\Delta$ and $J_{1}$ as depicted in equation (3.11). The tunnelling from the point to the giant graviton occurs only when the pair $\left(J_{1}, \Delta\right)$ is above the red line. Below the red line the point graviton is energetically favored and the tunnelling should be in the opposite direction.
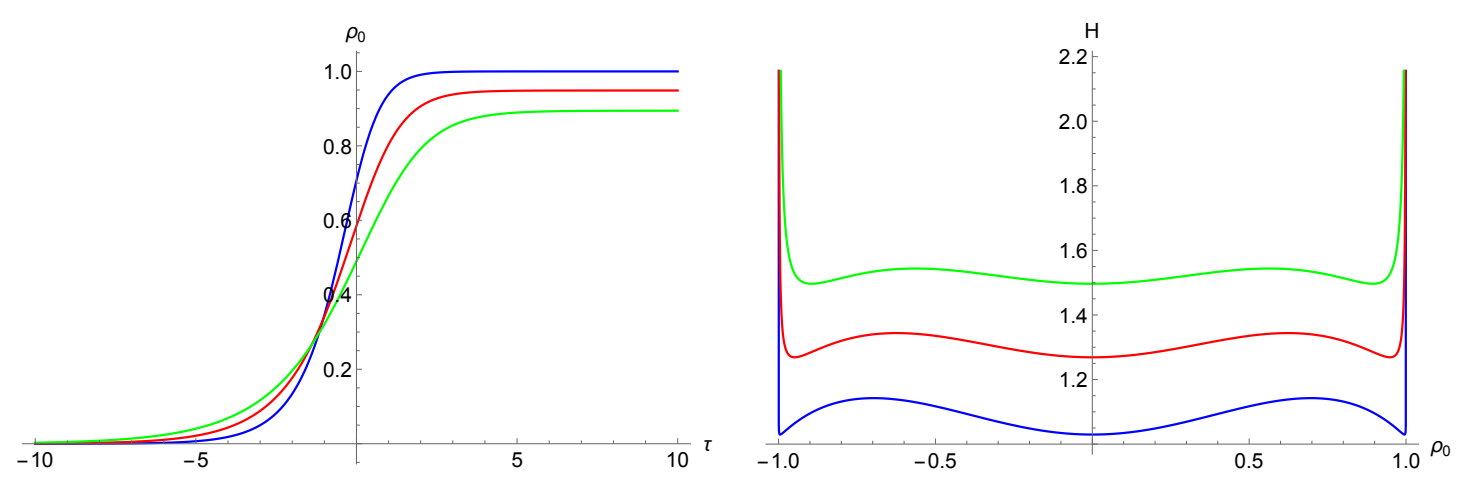

Figure 7. Left plot: instanton solution in the specific case that $\mathcal{W}=0$ in which the two graviton solutions are degenerate. The plot is for 3 different values of $J_{1}$. Right plot: Hamiltonian for the instanton solutions of the left plot. It is clear that when $\mathcal{W}=0$ the energies of the point and giant gravitons are equal. The correspondence between colors and values of $J_{1}$ is: Blue $\Rightarrow J_{1}=1.01$, Red $\Rightarrow J_{1}=1.1 \&$ Green $\Rightarrow J_{1}=1.2$

expanded giant graviton solution at $\tau \rightarrow-\infty$ to the zero size point graviton solution at $\tau \rightarrow+\infty$. From the moment that eventually, when increasing the deformation parameter, the energetically favored solution is the giant graviton, we are not interested in transitions from the giant to the point graviton solution.

On the right plot of figure 7 we plot the Hamiltonian of the special instanton solution. In that way a remarkable feature of the special solution is uncovered. When the constraint (3.11) is satisfied, the energies of the point graviton and of the giant graviton are equal. This situation is analogous to the instanton calculation in the undeformed case of $\mathcal{N}=4$ [3], where the energies of the point and the giant graviton were also equal. Here, tuning the relation between $\Delta$ and $J_{1}$, we are able to identify similar situations where the two solutions have the same energy. The important difference is that increasing the deformation parameter will eventually lead to an energetically favored giant graviton. 


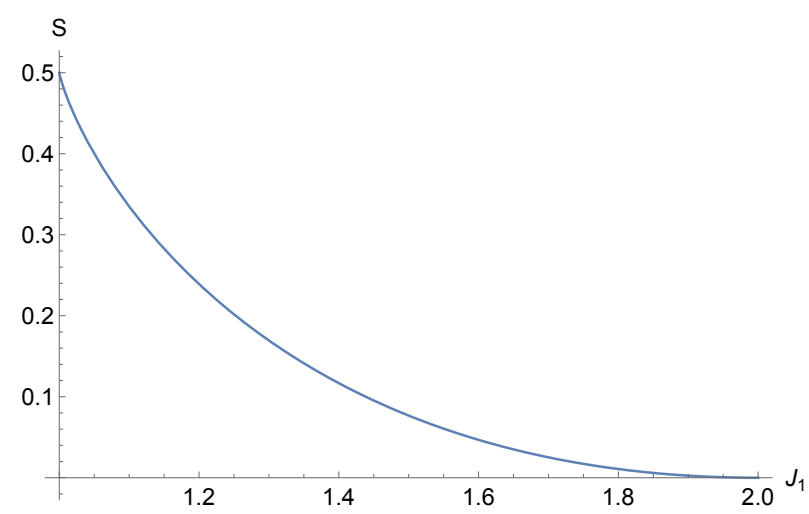

Figure 8. Total action as a function of $J_{1}$, in the special case of $\mathcal{W}=0$ from equation $(3.13) . J_{1}$ and $\Delta$ are related through equation (3.11).

The next important step is to calculate the total action of the special solution. Regularizing the Routhian as before in (3.10) but now integrating from $-\infty$ to $+\infty$ we are able to obtain an analytic result for the total action

$$
\begin{aligned}
\mathcal{S}=\int_{-\infty}^{+\infty} d \tau \Delta \mathcal{R} & =\frac{N}{L} \int_{0}^{L} \sqrt{2-\frac{J_{1}}{N}} d \rho \frac{2-\frac{\rho^{2}}{L^{2}}-\frac{J_{1}}{N}}{1-\frac{\rho^{2}}{L^{2}}} \frac{\rho}{L} \\
& =\frac{N}{2}\left[2-\frac{J_{1}}{N}+\left(\frac{J_{1}}{N}-1\right) \log \left(\frac{J_{1}}{N}-1\right)\right] .
\end{aligned}
$$

In figure 8 we plot the total action as a function of the angular momentum $J_{1}$, from where it is evident that it monotonically decreases. Thus, as $J_{1}$ ( or $J_{1} / N$, if we reinstate the number of colours) increases, the action decreases and the probability of tunnelling is increasing. This qualitative picture is also supported by the plot of the Hamiltonian (see the right plot of figure 7 ), where one can see that when $J_{1}$ increases, the difference between the extremum (minimum) of the point graviton and the nearby extremum (maximum), gets smaller. The potential becomes more shallow and as a result the probability of tunnelling becomes larger.

\section{Three-point function of two giant gravitons and a dilaton mode}

In this section, we will focus on the holographic calculation of the three-point correlation function involving two operators dual to the giant graviton and one operator dual to one of the dilaton modes. ${ }^{6}$ The result will provide the strong coupling prediction for the aforementioned three-point correlator. Similar holographic calculations involving giant magnons or spiky strings of the Schrodinger background have been performed recently in [23]. In addition, the holographic computation of heavy-heavy-light correlation functions with the heavy operators being the giant gravitons and the light operators being BPS states of the undeformed $A d S_{5} \times S^{5}$ background have been presented in [34].

\footnotetext{
${ }^{6}$ The method to calculate three-point correlators of the heavy-heavy-light type holographically, as well as several important results can be found in [28-33].
} 
In order to proceed with the holographic calculation we need a giant graviton solution that tunnels from one point of the boundary (this is one of the points where the dual field theory operator is situated) to another. To this end we construct a slight generalization of the giant graviton solution presented in section 2 . This reads

$$
\begin{aligned}
& T=\kappa \tau, \quad V=\nu \tau-\frac{\vec{X}_{0}^{2}}{4} \sin 2 \kappa \tau, \quad \vec{X}=\vec{X}_{0} \sin \kappa \tau, \\
& Z=Z_{0}, \quad \rho=\frac{\rho_{0}}{L} \quad \chi=\omega \tau,
\end{aligned}
$$

where the constants appearing in (4.1) are given by

$$
\begin{aligned}
& \kappa=\frac{1}{L}, \\
& \nu=\frac{Z_{0}^{2}}{L \mathcal{Q}}\left(\frac{J_{1}}{N}-1\right)^{2}, \quad \omega=\frac{2}{L \sqrt{\mathcal{Q}}}\left(1-\frac{J_{1}}{4 N}\right) \\
& \frac{\rho_{0}^{2}}{L^{2}}=2-\frac{J_{1}}{N}, \quad \mu^{2}=\frac{Z_{0}^{4}}{\mathcal{Q}}\left(\frac{J_{1}}{N}-1\right) \quad \text { with } \quad \mathcal{Q}=\frac{J_{1}^{2}}{4 N^{2}}+\frac{J_{1}}{N}-1 .
\end{aligned}
$$

Notice that we have turned on two additional coordinates of the Schrodinger part of the spacetime, namely $\vec{X}$. As can be seen from (4.1) this induces a modification of the $V$ coordinate, too. ${ }^{7}$

The next step is to use the following coordinate transformation in order to pass from the global to the Poincare coordinates [35]

$$
x^{+}=\tan T, \quad x^{-}=V-\frac{1}{2}\left(Z^{2}+\vec{X}^{2}\right) \tan T, \quad z=\frac{Z}{\cos T}, \quad \vec{x}=\frac{\vec{X}}{\cos T}
$$

and subsequently to perform an analytic continuation to the world-sheet time $\tau \rightarrow i \tau$ after which the solution takes the form

$$
\begin{aligned}
& \mathbf{x}=\frac{\mathbf{X}}{2} \tanh \kappa \tau, \\
& x^{-}=-\frac{i}{2}\left[Z_{0}^{2}-\frac{\mathbf{X}^{2}}{2 \mathbf{T}}\right] \tanh \kappa \tau+i \nu \tau, \quad \rho=\frac{\rho_{0}}{L} \quad \chi=i \omega \tau .
\end{aligned}
$$

This is the appropriate giant graviton solution travelling from one point of the boundary at $\tau=-\infty$ to another point at $\tau=+\infty$. In what follows, we will also need the on-shell value of the D3 brane Lagrangian which can be evaluated from (2.5). It reads

$$
\mathcal{L}_{\mathrm{on} \text { shell }}^{\mathrm{GG}}=i \frac{N}{4 \pi^{2} L \sqrt{\mathcal{Q}}}\left(1-\frac{J_{1}}{2 N}\right)^{2} \sin \theta .
$$

One may now define the following kinematic Schrodinger invariant

$$
v_{12}=-\frac{1}{2}\left(\frac{x_{12}^{2}}{t_{12}}+\frac{x_{23}^{2}}{t_{23}}-\frac{x_{13}^{2}}{t_{13}}\right)
$$

\footnotetext{
${ }^{7}$ Let us also mention that the three-point function calculation of this section can be performed analytically only for the special case where the deformation parameter $\Delta$ and the angular momentum $J_{1}$ are related through (3.11), that is when the point and the giant graviton are degenerate. The most general case can be treated in a similar way but the analogous to (4.2) expressions become very lengthy without gaining no further insight.
} 
where $t_{i j}=t_{i}-t_{j}$ and similarly $\vec{x}_{i j}=\left(\vec{x}_{i}-\vec{x}_{j}\right)$. In (4.6), $\left(\vec{x}_{1}, t_{1}\right)$ and $\left(\vec{x}_{2}, t_{2}\right)$ denote the positions of the heavy operator on the boundary while $\left(\vec{x}_{3}, t_{3}\right)$ the position of the dilaton mode. In what follows and in order to keep the expressions for the three-point correlator as simple as possible we take the time ordering of the operators to be as follows

$$
t_{3}<t_{1}<t_{2} \quad \& \quad t_{3} \rightarrow-\infty .
$$

As a result the invariant cross ratio simplifies to $v_{12}=-\frac{1}{2} \frac{x_{12}^{2}}{t_{12}}=\frac{1}{2} \frac{\mathbf{X}^{2}}{\mathbf{T}}$. One may use this last relation to rewrite, by taking into account that the expression for $Z_{0}$ becomes

$$
Z_{0}^{2}=\frac{v_{12}}{2}\left[1-\frac{E}{2 N \sqrt{\mathcal{Q}}}\right]^{-1}
$$

the analytically continued giant graviton solution (4.4) in terms of the invariant $v_{12}, \mathbf{X}$ and $\mathbf{T}=t_{2}-t_{1}$. It reads

$$
\begin{gathered}
\mathbf{x}=\frac{\mathbf{X}}{2} \tanh \kappa \tau, \quad x^{+}=i \frac{\mathbf{T}}{2} \tanh \kappa \tau, \quad z=\frac{\sqrt{\mathbf{T} v_{12}}}{2 \cosh \kappa \tau}\left[1-\frac{E}{2 N \sqrt{\mathcal{Q}}}\right]^{-1 / 2}, \\
x^{-}=i \nu \tau+\frac{i v_{12}}{2}\left[1-\frac{1}{2}\left[1-\frac{E}{2 N \sqrt{\mathcal{Q}}}\right]^{-1}\right] \tanh \kappa \tau, \quad \rho=\frac{\rho_{0}}{L}, \quad \chi=i \omega \tau . \quad
\end{gathered}
$$

The last piece of information needed is the bulk-to-boundary propagator of the light field, that is of the dilaton mode. In the limit (4.7) it takes the form [23]

$$
K\left(z, \vec{x}, t ; \vec{x}_{3}, t_{3}\right) \approx \frac{i\left(\frac{\mu M_{3}}{2}\right)^{\Delta_{3}-1} e^{-\frac{i}{2} \pi \Delta_{3}}}{\pi \Gamma\left(\Delta_{3}-2\right)}\left(\frac{z}{-t_{3}}\right)^{\Delta_{3}},
$$

where $M_{3}$ denotes the mass eigenvalue of the dilaton mode participating in the three-point correlator.

Now, we have all the ingredients in order to calculate the three-point correlator. As discussed, in some detail, in a similar calculation [23] the ratio of the three-point to the two-point correlation functions is given by

$$
\frac{G_{3}\left(\bar{x}_{1}, \bar{x}_{2}, \bar{x}_{3}\right)}{G_{2}\left(\bar{x}_{1}, \bar{x}_{2}\right)}=-\int d^{4} \xi e^{i M_{3} x^{-}(\tau, \sigma)} K\left(\bar{x}_{\text {classical }}(\tau, \sigma) ; \bar{x}_{3}\right) \mathcal{L}_{\text {on shell }}^{\text {classical }}
$$

Notice that the result for the three-point function takes the form of a vertex operator that corresponds to the light state integrated over the classical world-volume of the D3-brane which represents the giant graviton at the strong coupling regime. ${ }^{8}$

Plugging (4.5) and (4.10) and using the expressions for the solution (4.9) in (4.11) one may perform the integration over the three-sphere along which the D3-brane extends to obtain

$$
\frac{G_{3}}{G_{2}}=\frac{N v_{12}^{\frac{\Delta_{3}}{2}}}{\sqrt{\mathcal{Q}}} \frac{\left[1-\frac{J_{1}}{2 N}\right]^{2}}{\left[1-\frac{E}{2 N \sqrt{\mathcal{Q}}}\right]^{\frac{\Delta_{3}}{2}}} \frac{\left(\frac{M_{3}}{2}\right)^{\Delta_{3}-1} e^{-\frac{i}{2} \pi \Delta_{3}}}{2^{\Delta_{3}-2} \pi \Gamma\left(\Delta_{3}-2\right)}\left(\frac{\sqrt{t_{21}}}{-t_{3}}\right)^{\Delta_{3}} \frac{1}{L} \int_{-\infty}^{+\infty} \frac{e^{i M_{3} x^{-}(\tau, \sigma)}}{\left(\cosh \frac{1}{L} \tau\right)^{\Delta_{3}}} d \tau
$$

\footnotetext{
${ }^{8}$ The minus sign in front of the right hand side of (4.11) originates from the functional differentiation of the brane action with respect to the dilaton field (see (2.5)).
} 
The remaining $\tau$ integral can be approximated by

$$
\mathcal{I} \approx \frac{1}{L} \int_{-\infty}^{+\infty} \frac{e^{\frac{\alpha}{L} \tau}}{\left(\cosh \frac{1}{L} \tau\right)^{\Delta_{3}}} d \tau \quad \text { where } \quad \alpha=-\frac{v_{12} M_{3}}{2}\left[1+\frac{\mathcal{Q}-2\left(\frac{J_{1}}{N}-1\right)^{2}}{\frac{E}{N} \sqrt{\mathcal{Q}}-2 \mathcal{Q}}\right]
$$

The approximation is done in the exponential $e^{i M_{3} x^{-}(\tau, \sigma)}$ of (4.11) and consists of replacing the $\tanh (\tau / L) \approx \tau / L$ appearing in $x^{-}$. This is a legitimate approximation since the bulkto-boundary propagator in (4.11) has a peak around $\tau=0$ and falls off exponentially away from this value. As a result the integral can be evaluated to give

$$
\mathcal{I}=2^{\Delta_{3}-1} \mathrm{~B}\left[\frac{1}{2}\left(\Delta_{3}-\alpha\right), \frac{1}{2}\left(\Delta_{3}+\alpha\right)\right] .
$$

As discussed in [23] (see eq. (3.9)) the ratio of the three to the two-point correlator takes in the limit (4.7) the following form

$$
\frac{G_{3}}{G_{2}}=\left(\frac{\sqrt{t_{21}}}{-t_{3}}\right)^{\Delta_{3}} \tilde{F}\left(v_{12}\right)
$$

where $\tilde{F}\left(v_{12}\right)$ is an undetermined function of the Schrödinger invariant $v_{12}$ that can not be determined by conformal invariance. It reads

$$
\tilde{F}\left(v_{12}\right)=\frac{N v_{12}^{\frac{\Delta_{3}}{2}}}{\sqrt{\mathcal{Q}}} \frac{\left[1-\frac{J_{1}}{2 N}\right]^{2}}{\left[1-\frac{E}{2 N \sqrt{\mathcal{Q}}}\right]^{\frac{\Delta_{3}}{2}}} \frac{\left(\frac{M_{3}}{2}\right)^{\Delta_{3}-1} e^{-\frac{i}{2} \pi \Delta_{3}}}{2^{-1} \pi \Gamma\left(\Delta_{3}-2\right)} \mathrm{B}\left[\frac{1}{2}\left(\Delta_{3}-\alpha\right), \frac{1}{2}\left(\Delta_{3}+\alpha\right)\right] .
$$

Our calculation provides the strong coupling prediction for the three-point correlation function and is fully consistent with the three-dimensional conformal symmetry of the Schrödinger group of the theory.

\section{Conclusions}

The center of attention of the current paper is the construction and further study of a giant graviton configuration in the framework of the Schrödinger holography. On the gravity side the giant gravitons are described by a D3-branes that extend along the time direction of the $S_{c h}$ part of the geometry and wrap a three-sphere inside the internal space of the undeformed five-sphere. The main result of this paper is to confirm, in the original Schrödinger spacetime, the picture discovered previously in [10] for the case of the pp-wave limit of the Schrödinger geometry, namely that the giant graviton configuration becomes the energetically favored stable configuration compared to the point graviton one. This fact is remarkable by itself since in most of the literature the tendency is exactly the opposite, the point graviton is the ground state of the D3-brane. The aforementioned fact leads also to the possibility of tunnelling from the point to the giant graviton configuration. We calculate, explicitly, the instanton solution and its corresponding action which gives 
a measure of the tunnelling probability. Finally, we evaluate holographically the 3-point correlation function of two giant gravitons and one dilaton mode.

The giant graviton solution depends on the deformation parameter of the spacetime $\Delta$ and on the angular momentum $J_{1}$ along the sphere equator. We vary the value of the deformation parameter keeping the angular momentum fixed, either below or above the constant value $J_{1}=1$. In the former case and for non-zero values of the deformation parameter the degeneracy between the point and the giant graviton, that was present in the undeformed $\mathcal{N}=4$ case [3], is lifted in favor of the giant graviton. Moreover, there is a maximum value of the deformation parameter (e.g. $\Delta=1 / \sqrt{2}$ ) above which the point graviton solution ceases to be at a minimum of the potential and evetually disappears from the spectrum. Notice that both aforementioned features were present also in the analysis of the giant graviton solution in the pp-wave limit of the Schrödinger background [10]. Fixing the conjugate momentum above the constant value $J_{1}=1$ discloses the presence of a critical deformation parameter, where the energies of the point and the giant graviton solutions are equal. This in turn creates a whole class of solutions (for every value of $J_{1}$ between 1 and 2 there is a critical value of $\Delta$ between 0 and $1 / \sqrt{2}$ ) and the precise relation between $\Delta_{\text {crit }}$ and $J_{1}$ is given in equation (3.11). Below this critical value $\Delta<\Delta_{\text {crit }}$ the point graviton has less energy than the giant graviton while above, that is when $\Delta>\Delta_{\text {crit }}$ it is the giant graviton that is energetically favored. Notice that until now in the literature (see e.g. [8]) the mere presence of a deformation parameter in the geometry lifts a degeneracy between the point and the giant graviton solution. The fact that with a Schrödinger deformation such a degeneracy returns for a finite deformation is an interesting new feature and is related to the drastic changes of the dipole-deformation on the geometry, as well as on the field theory side.

Besides identifying the two graviton solutions (and motivated by the quantum mechanical expectation of state mixing) we focus on cases where the giant graviton is energetically favored with respect to the point graviton and construct the instanton solutions tunnelling from the point to the giant graviton. Plotting the total action as a function of the deformation parameter (see e.g. figure 4) we identify a monotonically decreasing behavior as the deformation parameter increases. This, in turn, means that the transition probability increases, something that is totally expected if one carefully examines the shape of the potential in figure 1. Indeed, as the deformation parameter increases the potential around the point graviton solution gets flattened and as result the probability of tunnelling from the point to the giant graviton increases. We give special attention to the case of the critical deformation parameter. In this case the two configurations have the same energy and the form of the potential resembles that of the $\mathcal{N}=4$ case of [3], but in the presence of a deformation. The calculation of the total action is analytic and reveals an increased tunnelling probability as the deformation parameter increases (or equivalently, due the relation that appears in equation (3.11), as the conjugate momentum increases).

Finally we focus our attention of the holographic calculation of the three-point correlation functions of the dilaton modes and two "heavy" operators, following the analysis that is detailed in [23]. In the case at hand, the "heavy" operator is the giant graviton solution and in particular the class of solutions where the deformation parameter acquires 
the critical value of equation (3.11). Moreover and in order for the classical solution to have the interpretation of a brane tunnelling between two boundary points (where the field theory operators will be located), the giant graviton solution in slightly generalized. The result of the computation is twofold: from one side we confirm the form of the correlator that is dictated by conformal invariance and from the other we specify the scaling function at strong coupling. This is a quantity that is not possible to fix using conformal symmetry.

There is a number of interesting directions which have not be explored in the context of Schrödinger holography. One of these is related to instanton calculations in the Schrödinger/null-dipole CFT duality. The analogous calculations for the marginal deformation of $\mathcal{N}=4$ called $\beta$-deformations and for the three parameter deformation of $\mathcal{N}=4$ [36] have been performed in [37] and [38], respectively. A second direction would be to focus on the field theory side of the duality and study the gauge invariant operators dual to the giant graviton configurations presented in this work. These should be obtained from the undeformed ones by introducing the appropriate star product among the $\mathcal{N}=4$ fields which will generate the dipole shift along one of the light-cone directions. Finally. it would be interesting to investigate which are the general features that a background should have so that the giant graviton solution is energetically favored compared to the point graviton. Our analysis points towards the direction that deforming the AdS part of the geometry is essential regarding this aspect.

\section{Acknowledgments}

The work of this project has received funding from the Hellenic Foundation for Research and Innovation (HFRI) and the General Secretariat for Research and Technology (GSRT), under grant agreement No 15425 .

Open Access. This article is distributed under the terms of the Creative Commons Attribution License (CC-BY 4.0), which permits any use, distribution and reproduction in any medium, provided the original author(s) and source are credited.

\section{References}

[1] J. McGreevy, L. Susskind and N. Toumbas, Invasion of the giant gravitons from Anti-de Sitter space, JHEP 06 (2000) 008 [hep-th/0003075] [INSPIRE].

[2] S.R. Das, A. Jevicki and S.D. Mathur, Vibration modes of giant gravitons, Phys. Rev. D 63 (2001) 024013 [hep-th/0009019] [inSPIRE].

[3] M.T. Grisaru, R.C. Myers and O. Tafjord, SUSY and Goliath, JHEP 08 (2000) 040 [hep-th/0008015] [INSPIRE].

[4] A. Hashimoto, S. Hirano and N. Itzhaki, Large branes in AdS and their field theory dual, JHEP 08 (2000) 051 [hep-th/0008016] [INSPIRE].

[5] M. Pirrone, Giants on deformed backgrounds, JHEP 12 (2006) 064 [hep-th/0609173] [INSPIRE].

[6] E. Imeroni and A. Naqvi, Giants and loops in beta-deformed theories, JHEP 03 (2007) 034 [hep-th/0612032] [INSPIRE]. 
[7] R. de Mello Koch, N. Ives, J. Smolic and M. Smolic, Unstable giants, Phys. Rev. D 73 (2006) 064007 [hep-th/0509007] [INSPIRE].

[8] S.D. Avramis, K. Sfetsos and D. Zoakos, Complex marginal deformations of D3-brane geometries, their Penrose limits and giant gravitons, Nucl. Phys. B 787 (2007) 55 [arXiv:0704 .2067] [INSPIRE].

[9] W.-H. Huang, Thermal giant graviton with non-commutative dipole field, JHEP 11 (2007) 015 [arXiv: 0709.0320] [inSPIRE].

[10] G. Georgiou and D. Zoakos, Giant gravitons on the Schrödinger pp-wave geometry, JHEP 03 (2020) 185 [arXiv : 2002.05460] [INSPIRE].

[11] J.M. Maldacena, The large $N$ limit of superconformal field theories and supergravity, Int. J. Theor. Phys. 38 (1999) 1113 [Adv. Theor. Math. Phys. 2 (1998) 231] [hep-th/9711200] [INSPIRE].

[12] J. Maldacena, D. Martelli and Y. Tachikawa, Comments on string theory backgrounds with non-relativistic conformal symmetry, JHEP 10 (2008) 072 [arXiv:0807.1100] [INSPIRE].

[13] C.A. Fuertes and S. Moroz, Correlation functions in the non-relativistic AdS/CFT correspondence, Phys. Rev. D 79 (2009) 106004 [arXiv:0903.1844] [INSPIRE].

[14] A. Volovich and C. Wen, Correlation functions in non-relativistic holography, JHEP 05 (2009) 087 [arXiv: 0903.2455] [INSPIRE].

[15] G. Georgiou and D. Zoakos, Giant magnons and spiky strings in the Schrödinger/dipole-deformed CFT correspondence, JHEP 02 (2018) 173 [arXiv:1712.03091] [INSPIRE].

[16] C.-S. Chu, G. Georgiou and V.V. Khoze, Magnons, classical strings and $\beta$-deformations, JHEP 11 (2006) 093 [hep-th/0606220] [INSPIRE].

[17] D. Zoakos, Finite size effects in classical string solutions of the Schrödinger geometry, JHEP 08 (2020) 091 [arXiv: 2006. 02285] [inSPIRE].

[18] C. Ahn and P. Bozhilov, Giant magnon-like solution in Sch ${ }_{5} x S^{5}$, Phys. Rev. D 98 (2018) 106005 [arXiv: 1711.09252] [INSPIRE].

[19] A. Golubtsova, H. Dimov, I. Iliev, M. Radomirov, R.C. Rashkov and T. Vetsov, More on Schrödinger holography, JHEP 08 (2020) 090 [arXiv:2004.13802] [INSPIRE].

[20] G. Georgiou, K. Sfetsos and D. Zoakos, String theory on the Schrödinger pp-wave background, JHEP 08 (2019) 093 [arXiv: 1906.08269] [INSPIRE].

[21] M. Guica, F. Levkovich-Maslyuk and K. Zarembo, Integrability in dipole-deformed $\mathcal{N}=4$ super Yang-Mills, J. Phys. A 50 (2017) 39 [arXiv:1706.07957] [InSPIRE].

[22] H. Ouyang, Semiclassical spectrum for BMN string in $S_{c h} \times S^{5}$, JHEP 12 (2017) 126 [arXiv: 1709.06844] [INSPIRE].

[23] G. Georgiou and D. Zoakos, Holographic three-point correlators in the Schrödinger/dipole CFT correspondence, JHEP 09 (2018) 026 [arXiv: 1806.08181] [INSPIRE].

[24] H. Dimov, M. Radomirov, R.C. Rashkov and T. Vetsov, On pulsating strings in Schrödinger backgrounds, JHEP 10 (2019) 094 [arXiv: 1903.07444] [INSPIRE].

[25] A. Golubtsova, H. Dimov, I. Iliev, M. Radomirov, R.C. Rashkov and T. Vetsov, Pulsating strings in Schr $_{5} \times T^{1,1}$ background, arXiv:2007.01665 [INSPIRE]. 
[26] H. Dimov, M. Radomirov, R.C. Rashkov and T. Vetsov, Holographic Fisher information metric in Schrödinger spacetime, arXiv:2009.01123 [INSPIRE].

[27] M. Alishahiha and O.J. Ganor, Twisted backgrounds, PP waves and nonlocal field theories, JHEP 03 (2003) 006 [hep-th/0301080] [INSPIRE].

[28] K. Zarembo, Holographic three-point functions of semiclassical states, JHEP 09 (2010) 030 [arXiv: 1008.1059] [INSPIRE].

[29] M.S. Costa, R. Monteiro, J.E. Santos and D. Zoakos, On three-point correlation functions in the gauge/gravity duality, JHEP 11 (2010) 141 [arXiv:1008.1070] [INSPIRE].

[30] G. Georgiou, Two and three-point correlators of operators dual to folded string solutions at strong coupling, JHEP 02 (2011) 046 [arXiv: 1011.5181] [INSPIRE].

[31] G. Georgiou, SL(2) sector: weak/strong coupling agreement of three-point correlators, JHEP 09 (2011) 132 [arXiv: 1107.1850] [INSPIRE].

[32] G. Georgiou, B.-H. Lee and C. Park, Correlators of massive string states with conserved currents, JHEP 03 (2013) 167 [arXiv:1301.5092] [INSPIRE].

[33] Z. Bajnok and R.A. Janik, Classical limit of diagonal form factors and HHL correlators, JHEP 01 (2017) 063 [arXiv: 1607. 02830] [INSPIRE].

[34] A. Bissi, C. Kristjansen, D. Young and K. Zoubos, Holographic three-point functions of giant gravitons, JHEP 06 (2011) 085 [arXiv:1103.4079] [INSPIRE].

[35] M. Blau, J. Hartong and B. Rollier, Geometry of Schrödinger space-times, global coordinates, and harmonic trapping, JHEP 07 (2009) 027 [arXiv: 0904.3304] [INSPIRE].

[36] S. Frolov, Lax pair for strings in Lunin-Maldacena background, JHEP 05 (2005) 069 [hep-th/0503201] [INSPIRE].

[37] G. Georgiou and V.V. Khoze, Instanton calculations in the beta-deformed AdS/CFT correspondence, JHEP 04 (2006) 049 [hep-th/0602141] [INSPIRE].

[38] C. Durnford, G. Georgiou and V.V. Khoze, Instanton test of non-supersymmetric deformations of the $A d S_{5} \times S^{5}$, JHEP 09 (2006) 005 [hep-th/0606111] [INSPIRE]. 